\title{
Comparison of the Linear Approximations for the Dynamic Security Region of Network- reduction Power Systems
}

\author{
Ancheng Xue, Wei Hu, Shengwei Mei, Member, IEEE, Yixin Ni, Senior Member, IEEE, Felix F. Wu, \\ Fellow, IEEE, and Qiang Lu, Fellow, IEEE
}

\begin{abstract}
The Dynamic Security Region (DSR) of bulk power system has been accepted more and more in recent years for providing plenty of security information and good prospect in online application. This paper compares three linear approximations for the dynamic security region of networkreduction power systems. The three linear approximations are the Q-linear approximation based on the quadratic approximation of stability region, the L-linear approximation based on the linear approximation of stability region and the LOlinear approximation based on the invariant assumption of the normal vector for the boundary of the stability region corresponding to different control variable. The three linear approximations are all obtained with a same critical point lying just on the boundary of dynamic security region. The critical point is searched with numerical simulation. The accuracy of the three linear approximations is compared, using the linear approximation obtained with the curve fitting approach or the actual boundary of DSR searched as the benchmark. Simulation results in IEEE 3-machine 9-bus system and 10-machine 39-bus New England system show that all the three linear approximations display fairly accurate estimation. Furthermore, from the computational viewpoint, the L-linear and the LO-linear method are two alternative choices to approximate the dynamic security region.
\end{abstract}

Index Terms-Dynamic Security Region; Transient Stability; Stability Region; Direct Method; Linear Approximation

\section{INTRODUCTION}

$\mathrm{T}$ ransient stability has always been one of the most important analyses in power system engineering. Transient stability is the ability of the power system to maintain synchronism after a fault such as short circuit. Significant advances have been made in recent years in the

This work is supported by the National Science Foundation of China (No.50377018, No.50595411), the National Key Basic Research Special Fund of China (G2004CB217900) and the Research Grant Council of HKSAR(HKU7176/03E).

Ancheng Xue is with the State Key Lab of Power System, Dept. of Electrical Engineering, Tsinghua University, Beijing 100084, P. R. China and the Graduate School at Shenzhen, Tsinghua University, Shenzhen 518055, P. R. China. (e-mail: xueac97@mails.tsinghua.edu.cn).

Wei Hu, Shengwei Mei and Qiang Lu are with the State Key Lab of Power System, Dept. of Electrical Engineering, Tsinghua University, Beijing 100084, P. R. China. (e-mail: huwei@mail.tsinghua.edu.cn, meishengwei@mail.tsinghua.edu.cn, luqiang@mail.tsinghua.edu.cn).

Yixin Ni and Felix F. Wu are with the University of Hong Kong, Hong Kong SAR, P. R. China. (e-mail: yxni@eee.hku.hk, ffwu@eee.hku.hk).
Direct Methods for power system transient stability analysis [1-7]. However, due to both the fault and its clearing time are fixed, the power system transient stability can be uniquely determined by some system control variable. Thus it is useful to clarify the set of control variable-dynamic security region - each of which indicate that the power system can remain transient stable against a certain fault.

The concept of dynamic security region was introduced many years ago [8]. It has been made practical by a series of modifications and enhancements in the last couple of years [914]. Ref. [10] observed that the practical DSR is surrounded by the vertical hyper-planes that are upper and lower limits of every bus injection, and one or several the hyper-planes that describe critical points of transient stability on power injection space. To the latter, Ref [11] constructed it by the least square curve fitting approach, which uses a lot of critical points searched by numerical simulation, furthermore, it gave out the practical DSR of the Central China power system. However, it is impractical to obtain DSR by numerical simulation in the large power system. Analytical methods to direct calculate the DSR have been proposed in $[12,13]$. By assuming that planes, tangent to the boundaries of transient stability region at the CUEPs, are parallel under different critical power injections (i.e. the normal vector of the planes are being the same constant corresponding to different control variable), ref. [12] proposed a practical analytic expression of the boundary of the DSR respected to the critical transient stability. Furthermore, by extending the parallel character to the exit point of the fault-on trajectory, ref. [13] developed a direct method for rapidly determining the hyper-planes corresponding to critical transient stability region, based on the transient energy function. Recently, ref. [14] presents an implicit expression for the local boundary of the DSR based on the implicit expression of stability region, and subsequently, some approximations for the DSR are derived with the approximation for the stability region and sensitivities.

It is reported [12-14] that if we obtain one critical point just lying on the boundary of dynamic security region as the value of the control variable where the proposed approximation method is applied, then we can obtain fairly good approximation for the local boundary of DSR. In this paper, we compare three linear approximations for the dynamic security region, after obtaining one critical point on 
the boundary of DSR. The three linear approximations are the Q-linear approximation based on the quadratic approximation of stability region, the L-linear approximation based on the linear approximation of stability region and the L0-linear approximation based on the invariant assumption of the normal vector of the boundary of the stability region corresponding to different control variable. In the comparison, we use the linear approximation of the DSR obtained by the least square curve fitting method as the benchmark. And furthermore, we compare the angle of between the normal vectors of the linear approximations and benchmark, and the coefficient error of the approximation in uniform displaying form. Additionally, for the DSR in low dimension, we use the figure to directly compare the approximation with the actual boundary searched by numerical simulation. The simulations in IEEE 3-machine 9-bus system and 10-machine 39-bus New England system show that all the three approximations display fairly accurate estimation. Furthermore, from the computational viewpoint, the L-linear and the L0-linear method are two alternative choices to approximate the dynamic security region.

The remainders of the paper are organized as follows: after obtaining the implicit expression for the dynamic security region (DSR) in Section II, we present three linear approximations for the DSR in Section III. We then discuss the problem in approximations and comparison in Section IV. The approximations are compared using IEEE 3-machine 9bus system and 10-machine 39-bus in Section V. Finally, a summary is provided in Section VI.

\section{Power System Transient Stability and Dynamic SECURITY REGION}

\section{A. Power System Model}

Consider a power system with generators and loads interconnected together by a transmission network. The system dynamics is assumed to be represented by a differential equation

$$
\dot{x}=f(x, u)
$$

where $x \in R^{n}$ are the state variables, and $u \in R^{m}$ are the control variables. Due to power flow equations of the transmission network, the electromechanical interaction of a power system for stability analysis is typically represented by a differential-algebraic equation instead. Additional assumption is therefore necessary for the model introduced in (1) to be valid for stability analysis. One is to assume that the loads are all constant impedance loads. Another one is to assume that the structural preserving model [7] is used.

For example, if the classical model of a power system is used for transient stability analysis, then for a power system consisting of $n_{g}$ generators with the loads modeled as constant impedances, the dynamics of the $k$-th generator can be written with the usual notation as:

$$
\begin{aligned}
& \dot{\delta}_{k}=\omega_{0} \omega_{k} \\
& 2 H_{k} \dot{\omega}_{k}=P_{m k}-P_{e k}-D_{k} \omega_{k}
\end{aligned} k=1, \cdots, n_{g}
$$

where $\omega_{0}=2 \pi f_{B}, \delta_{k}$ and $\omega_{k}$ are the rotor angle and speed of machine $k ; D_{k}$ and $H_{k}$ are damping ratio and inertia constant of machine $k, P_{m k}$ and $P_{e k}$ are the mechanical power and the electrical at machine $\# k$;

$$
P_{e k}=\left\{E_{k}^{2} G_{k k}+E_{k}\left(\sum_{j \neq k}^{n_{g}} E_{j}\left(G_{k j} \cos \delta_{k j}+B_{k j} \sin \delta_{k j}\right)\right)\right\}
$$

where $\delta_{k j}=\delta_{k}-\delta_{j}, E_{k}$ is the constant voltage behind direct axis transient reactance of machine $\# k$., $\boldsymbol{Y}=\left(G_{i j}+j B_{i j}\right)_{n \times n}$ is the reduced admittance matrix.

If, furthermore, as usual, uniform damping is assumed, i.e. $d_{0}=D_{k} / 2 H_{k}\left(k=1, \cdots, n_{g}\right)$, then using the $n_{g}-t h$ machine as the reference, (2) can be transformed into the form of (1) as follows:

$$
\begin{aligned}
\dot{\delta}_{k n_{g}}= & \omega_{0} \omega_{k n_{g}} \\
\dot{\omega}_{k n_{g}}= & -d_{0} \omega_{k n_{g}}+\left(P_{m k}-P_{e k}\right) / 2 H_{k} \quad k=1, \cdots, n_{g}-1 \\
& -\left(P_{m n_{g}}-P_{e n_{g}}\right) / 2 H_{n_{g}}
\end{aligned}
$$

In such a case, the state variables will be $x=\left(\delta^{T}, \omega^{T}\right)^{T} \quad, \quad$ where $\quad \delta=\left(\delta_{1 n_{g}}, \cdots \delta_{n_{g}-1, n_{g}}\right)^{T}$, $\omega=\left(\omega_{1, n_{g}}, \cdots, \omega_{n_{g}-1, n_{g}}\right)^{T}$ and the control variables could be $u=\left(P_{m 1}, \cdots, P_{m n_{g}}\right)^{T}$.

\section{B. Transient Stability and Dynamic Security Region}

Transient stability is the ability of the power system to maintain synchronism after a fault such as short circuit. Mathematically, the power system suffered from a fault has three stages: the pre-fault, fault-on and post-fault stage.

At the pre-fault stage, the system is operated at a stable equilibrium point $x_{0}(u)$ of the pre-fault system

$$
\dot{x}=F_{1}(x, u) t<0
$$

At time $t=0$, the system undergoes a fault that results in a structural change in the system. Suppose the fault is cleared at time $t=t_{F}$. Then during the fault-on stage, the system is governed by a fault-on dynamics described by:

$$
\dot{y}=F_{2}(y, u), y(0)=x_{0}, y(t)=\phi\left(t, x_{0}, u\right), 0 \leq t<t_{F}
$$

Once the fault is cleared, the system is henceforth governed by a post-fault dynamics described by the following differential equation (6). The initial condition of the post-fault system is the state of the fault-on system at fault clearing, $\phi\left(t_{F}, x_{0}, u\right)$. Notice that since the clearing time is given and $x_{0}$ is a function of $u$, the system state at the time of clearing is really only a function of $\mathrm{u}$, we therefore write $\phi(u)=\phi\left(t_{F}, x_{0}, u\right)$. The post-fault dynamics is described by:

$$
\dot{z}=f(z, u), z\left(t_{F}\right)=y\left(t_{F}\right)=\phi(u), t \geq t_{F}
$$

Assuming the post-fault system has a (asymptotically) 
stable equilibrium point $z_{s}(u)$, then the transient stability analysis is to determine whether the initial point of the postfault trajectory, $\phi(u)$, is located inside the stability region of the equilibrium point $z_{s}(u), V\left(z_{s}(u)\right)$. Furthermore, as the setting of the control variables $u$ completely determines the transient stability of the system. We therefore can define a region in the space of control variables $u$ in which the system is transiently stable and called it the dynamic security region of the power system (with respect to a given fault). Mathematically, the dynamic security region (DSR) can be described as follows:

$$
\Omega_{d}=\left\{u: \quad \phi(u) \in V\left(z_{s}(u)\right)\right\}
$$

In the power system transient stability analysis, the concept of Controlling Unstable Equilibrium Point (CUEP) has been well recognized [1-6]. The CUEP of a certain fault is the unstable equilibrium point whose stable manifold is crossed by the continuous faulted trajectory of the fault [6]. Furthermore, the stable manifold of a controlling unstable equilibrium point $z_{e}$ can be described as follows [15]:

$$
\begin{aligned}
W^{s}\left(z_{e}\right)= & \left\{z \mid h(z, u)=0, \operatorname{rank}\left[\frac{\partial h(z, u)}{\partial z}\right]=1,\right. \\
& \text { s.t. } \left.\frac{\partial h(z, u)}{\partial z} f(z, u)=\mu h(z, u), h\left(z_{e}, u\right)=0\right\}
\end{aligned}
$$

where $\mu$ is the unstable eigen-value at the unstable equilibrium point $z_{e}$.

With the concept of the CUEP, the local boundary of dynamic security region that is of interest to the study of transient stability can therefore be written locally as[14]:

$$
\left.\partial \Omega_{d}\right|_{\text {local }}=\{u: \quad h(\phi(u), u)=0\}
$$

\section{LINEAR APPROXIMATIONS FOR THE LCOAL BOUNDARY OF DYNAMIC SECURITY REGION}

\section{A. Approximation for Stability Region}

The quadratic approximation of the unstable manifold $h(z, u)$ at $z_{e}$ in (8) can be expressed as:

$$
h_{Q}(z, u)=\eta(u)^{T}\left(z-z_{e}\right)+\left(z-z_{e}\right)^{T} Q(u)\left(z-z_{e}\right)
$$

where the coefficient $\eta$ and $Q$ of the quadratic expression can be determined as follows:

The coefficient vector $\eta \in R^{n}$ of the linear term is the left eigenvector associated with the eigenvalue $\mu$ of the Jacobian matrix $J(u)$ of $f$ at the equilibrium point $z_{e}$, i.e.:

$$
J(u)^{T} \eta(u)=\mu(u) \eta(u), \quad \eta(u)^{T} \eta(u)=1
$$

The coefficient matrix $Q$ of the quadratic term is the solution of the Lyapunov equation:

$$
C(u) Q(u)+Q(u) C(u)^{T}=H(u)
$$

where $C(u)=\mu(u) I / 2-J(u)^{T} ; I$ is the $n \times n$ identical matrix, and $H(u)=\left[\sum_{i=1}^{n} \eta_{i}(u) H_{i}(u)\right] ; H_{i}(u)$ is the Hessian matrix of $f_{i}$.

\section{B. Linear Approximations for Local Boundary of DSR}

Let us assuming that we have obtained a critical point lying on the boundary of the dynamic security region, i.e. $h\left(\phi\left(u_{0}\right), u_{0}\right) \approx 0$. Then, we are interested in finding the local boundary of the dynamic security region or its approximations, with the information at the critical point $u_{0}$. As the operating condition $u$ changes from $u_{0}$ to $u=u_{0}+\Delta u$, $\phi(u), z_{e}(u)$ and $h(z, u)$ all change, hence the equation of the local boundary of DSR $h(\phi(u), u)=0$ as well. Let us assume we have calculated the all the relevant quantities at $u_{0}$, i.e., $z_{e}\left(u_{0}\right), \phi\left(u_{0}\right), J\left(u_{0}\right), \mu\left(u_{0}\right), \eta\left(u_{0}\right), Q\left(u_{0}\right)$, $\eta\left(u_{0}\right)$ and the corresponding sensitivities. Then we can use the sensitivities to approximate the relevant quantities as follows:

$$
\begin{gathered}
z_{e}(u) \approx z_{e}\left(u_{0}\right)+Z\left(u_{0}\right) \cdot \Delta u \\
\eta(u) \approx \eta\left(u_{0}\right)+E\left(u_{0}\right) \cdot \Delta u \\
Q(u) \approx Q\left(u_{0}\right)+\sum_{i=1}^{m} Q_{i}\left(u_{0}\right) \Delta u_{i} \\
\phi(u) \approx \phi\left(u_{0}\right)+\Phi\left(u_{0}\right) \cdot \Delta u
\end{gathered}
$$

where $\quad Z(u)=\frac{\partial z_{e}(u)}{\partial u} \quad, \quad E(u)=\frac{\partial \eta(u)}{\partial \eta} \quad$ and $Q_{i}(u)=\frac{\partial Q(u)}{\partial u_{i}} \quad, \quad \Phi(u)=\frac{\partial \phi(u)}{\partial u} \quad$ is the trajectory sensitivities.

Substituting the approximate expressions (13)-(16) into the equation in (10), we obtain a linear approximation to the section of the boundary of the dynamic security region (9) based on the quadratic approximation for the stability region (Q-linear approximation) as follows:

$$
h_{Q L}(\phi(u), u)=C_{0}+C_{1}\left(u-u_{0}\right)=0
$$

The coefficients of the above quadratic approximations are, $C_{0}=h_{Q}\left(\phi\left(u_{0}\right), z_{e}\left(u_{0}\right)\right)$

$$
\begin{aligned}
C_{1} & =\eta\left(u_{0}\right)^{T} D\left(u_{0}\right)+d\left(u_{0}\right)^{T} E\left(u_{0}\right)+d\left(u_{0}\right)^{T} Q\left(u_{0}\right) D\left(u_{0}\right) \\
& +\left[d\left(u_{0}\right)^{T} Q_{1}\left(u_{0}\right) d\left(u_{0}\right), \cdots, d\left(u_{0}\right)^{T} Q_{m}\left(u_{0}\right) d\left(u_{0}\right),\right]
\end{aligned}
$$

where $d\left(u_{0}\right)=\phi\left(u_{0}\right)-z_{e}\left(u_{0}\right)$ is the difference between the system state at the time of fault clearing $\phi\left(u_{0}\right)$ and the CUEP $z_{e}\left(u_{0}\right)$, and $D\left(u_{0}\right)=\Phi\left(u_{0}\right)-Z\left(u_{0}\right)$ is its sensitivity with respect to $u$.

If we start directly from a linear approximation to the stability boundary: 


$$
h_{L}(z, u)=[\eta(u)]^{T}\left(z-z_{e}(u)\right)
$$

and substituting (13)-(16) into the equation in (18), we get a linear approximation to the section of the boundary of the dynamic security region (9) based on the linear approximation for the stability region (L-linear approximation) as follows:

$$
h_{L L}(\phi(u), u)=L_{0}+L_{1}\left(u-u_{0}\right)=0
$$

where $\quad L_{1}=\eta\left(u_{0}\right)^{T} D\left(u_{0}\right)+d\left(u_{0}\right)^{T} E\left(u_{0}\right)$ and

$$
L_{0}=h_{L}\left(\phi\left(u_{0}\right), u_{0}\right) \text {. }
$$

If we assume the normal vector of the boundary of the stability region being constant under different control variable $u$, i.e. if we start directly from a linear approximation to the stability boundary:

$$
h_{L^{*}}(z, u)=\left[\eta\left(u_{0}\right)\right]^{T}\left(z-z_{e}(u)\right)
$$

and substituting (13)-(16) into (20), we get a linear approximation to the section of the boundary of the dynamic security region (L0-linear approximation) as follows:

$$
h_{L 0}(\phi(u), u)=L_{0}+L_{10}\left(u-u_{0}\right)=0
$$

where $L_{10}=\eta\left(u_{0}\right)^{T} D\left(u_{0}\right)$.

Remark 3.1: The computation of the sensitivity in (13)-(16) can be found in ref. [14].

\section{Algorithm AND Discussion}

\section{A. Algorithms for Linear Approximations}

To obtain linear approximations in section 3.2, we should first obtain one critical point just lying on the boundary of dynamic security region, i.e., the value $u_{0}$ satisfying the equation $h\left(\phi\left(u_{0}\right), u_{0}\right)=0$. To do this, we first fix a direct in the control variable space, and then search the critical point along this direction with the numerical simulation, i.e. the point $u_{0}$ at which the critical clearing time of the system is equal to the fixed clearing time. With the above statement, we can have the general algorithm for the linear approximations of the local boundary of dynamic security region as follows:

Step 1:Determine the given fault, the clearing time $t_{F}$ and the control variable $u$;

Step 2: Select one practical direction in the control variable space, and then search for the critical control variable $u_{0}$ under which the critical clearing time is $t_{F}$;

Step 3: Calculating the state at clearing time $\phi\left(u_{0}\right)$ and the trajectory sensitivity $\Phi\left(u_{0}\right)$;

Step 4: Calculate the CUEP of the system $z_{e}\left(u_{0}\right)$ and the corresponding sensitivity $Z\left(u_{0}\right)$;

Step 5: Calculate the linear coefficient $\eta\left(u_{0}\right)$ and the corresponding sensitivity $E\left(u_{0}\right)$;

Step 6: Calculate the coefficient matrix $Q\left(u_{0}\right)$ and the corresponding sensitivity $Q_{i}\left(u_{0}\right)$;

Step 7: Obtain the linear approximation (17), (19) and
(21) of DSR;

Step 8: Convert the linear approximations to the uniform equation $a^{T} u=1\left(a=\left(a_{1}, \cdots, a_{m}\right)^{T}\right)$, as the PDSR[16-18].

\section{B. Comparison of the Linear Approximations}

In the computation viewpoint, as the three linear approximations all pass through the same critical point, thus the computation cost for searching the critical point is same. Furthermore, it is clear that the L0-linear approximation only needs the trajectory sensitivity (sensitivity of the state at the clearing time), and the L-linear approximation need the trajectory sensitivity and sensitivities of eigenvector, while the Q-linear approximation needs coefficients matrix of quadratic term and the corresponding sensitivities obtained by the Lyapunov equation, besides the trajectory sensitivity and sensitivities of eigenvector. Thus, the L0 linear approximation costs least computational burden among the three approximations, thus the L0-linear and L-linear approximation are more efficient than the Q-linear approximation as absence of solving Lyapunov equation.

To compare the accuracy of the approximations, we take the linear approximation, $b^{T} u=1,\left(b=\left(b_{1}, \cdots, b_{m}\right)^{T}\right)$, which obtained by the least square curve-fitting approach with the point searched around the critical point, as the benchmark. In comparison, we use the following two indexes to identify the difference between the proposed linear approximations and the benchmark linear approximation.

Index 1: Angle difference. We compare the accuracy of approximation using the angle difference between the normal vector of the proposed linear approximations and benchmark, i.e., $\theta=\cos ^{-1}(a \cdot b /(\|a\| \cdot\|b\|))$.

Index 2: Coefficient difference. We compare the accuracy of approximation using the norm of the coefficient distance between the normal vector of the proposed linear approximations and the benchmark, i.e., $C D_{b a}=\|a-b\|_{2}$.

Furthermore, for the DSR in the low dimension, we use the figure to directly compare the accuracy of linear approximation with the actual boundary of DSR searched by numerical simulation approach.

\section{Simulation Results}

\section{A. The 3-Generator 9-Bus System}

The 3-generator 9-bus IEEE test system [17, p.38] is used to compare the proposed methods. Classical models of generators with uniform damping $d_{0}=0.1661$ and network reduction procedure are used to derive the differential equation (1) of the model. We assume that the generator powers are the control variables. Therefore, the dimension of $u$ is equal to three.

A three-phase fault occurs at bus 7 on the line between nodes 7 and 5 . The fault is cleared at 0.10 second after the fault occurrence. If we choose one critical point on the boundary of the dynamic security region, e.g. a critical point 
obtained by only increasing the mechanical power of generator at bus 3, then we can obtain the coefficients of the least square linear approximation(LS), L0-linear approximation(L0), L-linear approximation(LL), Q-linear approximation(QL) in the uniform equation and the comparison results in Table I.

TABLE I

RESULTS OF DSR APPROXIMATIONS AND COMPARISON FOR FAULT 7-5

\begin{tabular}{ccccccc}
\hline \multirow{2}{*}{ Type } & \multicolumn{3}{c}{ Coefficients } & & \multicolumn{2}{c}{ Difference } \\
\cline { 2 - 4 } & $a_{1}$ & $a_{2}$ & $a_{3}$ & & Angle(deg) & Coefficient \\
\hline LS & -0.2418 & 0.3544 & 0.3422 & & - & - \\
L0 & -0.2259 & 0.2997 & 0.3865 & & 7.53 & 0.0721 \\
LL & -0.2427 & 0.3576 & 0.3392 & & 0.46 & 0.0045 \\
QL & -0.2567 & 0.4063 & 0.2994 & & 6.86 & 0.0690 \\
\hline
\end{tabular}

Simulations in Table I display that the angle differences between the normal vector of the least square linear approximation (LS) and the normal vectors of L0-linear approximation (L0), L-linear approximation(LL), Q-linear approximation(QL) are very small and the coefficient difference are also very small. This indicates that the proposed three linear approximations all give out fairly good results for the DSR. Furthermore, from the viewpoint of computation, the Q-linear approximation requires the computation cost on solving the Lyapunov equation, while the L-linear and L0 linear approximation does not. Thus, the L0 and L-linear approximation are two more practical choices to approximate the DSR.

Furthermore, we give out the projected map of the dynamic security region for the fault 7-5 in Fig 1.

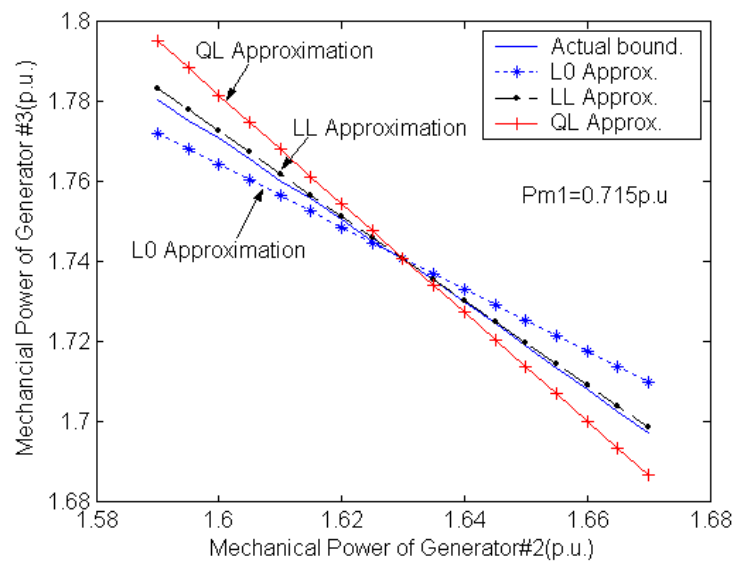

Fig. 1 DSR Linear Approximation of a 3M9B system to Fault 7-5

The actual boundary in Fig.1 is obtained by searching method. Fig. 1 shows that the L-linear approximation gives out the best estimation of the dynamic security for this line fault 7-5. This consists with the results of angle difference, coefficient difference in Table I. This also suggests that though the L0 approximation requires the least computation cost, but it could not replace the L-linear approximation in the accuracy.

\section{B. 10 Generator 39-Bus New England System}

The simulation results presented in this subsection are based on a 10-generator 39-bus New England system [1]. The system is modeled by the network-reduction model with classical generators having the uniform damping $d_{0}=0.2$.

A three-phase fault occurs at bus 17 on the line between nodes 17 and 16 . The fault is cleared at 0.10 second after the fault occurrence. The critical point is obtained only increased the mechanical power of generator at bus \#36, then we can obtain the coefficients of the least square linear approximation(LS), L0-linear approximation(L0), L-linear approximation(LL), Q-linear approximation(QL) in uniform equation in the Table II and the comparison results in the Table III.

TABLE II

COEFFICIENT OF DSR APPROXIMATIONS FOR LINE FAULT 17-16

\begin{tabular}{ccccc}
\hline \multirow{2}{*}{ Type } & \multicolumn{4}{c}{ Approximations Coefficients $\left(10^{-2}\right)$} \\
\cline { 2 - 5 } & L0 & LL & QL & LS \\
\hline$a_{1}$ & -1.0956 & -0.5288 & -1.3228 & -0.5867 \\
$a_{2}$ & -0.8772 & 0.2767 & -1.7181 & 0.0941 \\
$a_{3}$ & -0.4023 & 0.6540 & -1.3412 & -0.0243 \\
$a_{4}$ & 4.5992 & 3.6959 & 5.1565 & 4.3917 \\
$a_{5}$ & 5.6224 & 4.6664 & 6.4695 & 4.7052 \\
$a_{6}$ & 5.2215 & 4.1994 & 5.8420 & 4.9021 \\
$a_{7}$ & 5.9825 & 4.7585 & 6.8154 & 5.6130 \\
$a_{8}$ & -1.0433 & -0.3344 & -1.3848 & -0.8487 \\
$a_{9}$ & -1.2455 & -0.2715 & -1.8461 & -1.8737 \\
$a_{10}$ & -0.9241 & -0.9818 & -0.8919 & -0.3640 \\
\hline
\end{tabular}

TABLE III

COMPARISON RESULTS FOR FAULT 17-16

\begin{tabular}{ccc}
\hline \multirow{2}{*}{ Type } & \multicolumn{2}{c}{ Difference } \\
\cline { 2 - 3 } & Angle(deg) & Coefficient \\
\hline L0 & 8.32 & 0.0179 \\
LL & 11.87 & 0.0233 \\
QL & 11.60 & 0.0349 \\
\hline
\end{tabular}

Simulations results in Table II and Table III show that the angle differences and the coefficient difference between the least square linear approximation (LS) and L0-linear approximation (L0), L-linear approximation(LL), Q-linear approximation(QL) are very small. This indicates the proposed three linear approximations all give out fairly good results for the DSR.

Furthermore, if only some machines' the mechanical power can be changed, e.g., assuming only three machines, the machine at bus \#34, bus \#35 and bus \#36 are controllable. Then we can obtain the coefficient of the linear approximations in the uniform equation and the comparison results in Table IV.

TABLE IV

RESULT OF DSR APPROXIMATIONS FOR FAULT 17-16 IN LOW DIMENSION

\begin{tabular}{lcccccc}
\hline \multirow{2}{*}{ Type } & \multicolumn{3}{c}{ Coefficients $\left(10^{-2}\right)$} & & \multicolumn{2}{c}{ Difference } \\
\cline { 2 - 4 } \cline { 6 - 7 } & $a_{4}$ & $a_{5}$ & $a_{6}$ & & Angle(deg) & Coefficient \\
\hline LS & 5.3137 & 4.8283 & 5.6500 & & - & - \\
L0 & 5.2756 & 4.8994 & 5.6135 & & 0.55 & 0.0009 \\
LL & 5.4225 & 4.8798 & 5.5295 & & 1.06 & 0.0017 \\
QL & 5.3447 & 4.8262 & 5.6304 & & 0.23 & 0.0004 \\
\hline
\end{tabular}


In the case of machines at bus \#34, bus \#35 and bus \# 36 are controllable, we can obtain the projected DSR with the mechanical power of machine at bus \#34 being 5.08 p.u., shown in Fig. 2. The actual boundary in Fig. 2 is obtained by searching method. Fig. 2 shows that the proposed three linear approximations all give out fairly good results for the DSR.

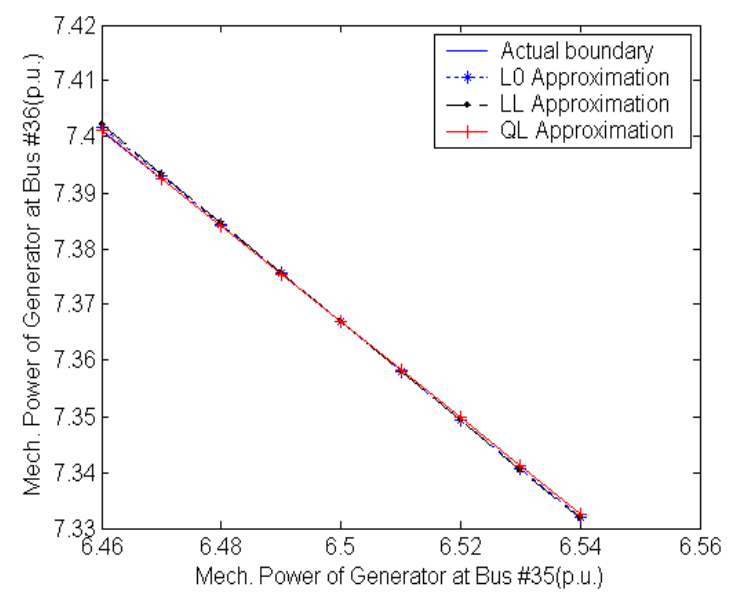

Fig. 2 DSR Approximation of a 10M39B system for Fault 17-16

\section{CONCLUSIONS}

This paper compares the three linear approximations, Qlinear approximation the L-linear approximation and the L0linear approximation for the dynamic security region of power system with network reduction model. The linear approximations used in the comparison are obtained with a critical point searched with numerical simulation. The simulations in IEEE 3-machine 9-bus system and 10-machine 39-bus New England system show that all the three approximations display fairly accurate estimation. Furthermore, from the computational viewpoint, the L-linear and the L0-linear method are two alternative choices to approximate the dynamic security region.

\section{REFERENCES}

[1] M. A. Pai, Energy function analysis for power system stability. Boston: Kluwer Academic Publishers, 1989.

[2] A. A. Fouad, V. Vittal. Power system transient stability analysis using the transient energy function method, Englewood Cliffs, N. J. : Prentice Hall, 1992.

[3] H. D. Chiang, C. C. Chu, and G. Cauley, "Direct Stability Analysis of Electric Power Systems Using Energy Functions: Theory, Applications and perspective", Proceedings of the IEEE, Vol. 83, No.11, pp.14971529, Nov. 1995.

[4] Xue Y, Van Custem T, Ribbens-Pavella M. "Extended equal area criterion justifications, Generalizations, applications." IEEE Transactions on Power Systems, Vol. 4, No. 1, pp. 44-52, 1989.

[5] H. D. Chiang, M. W. Hirsch, F. F. Wu, "Stability regions of nonlinear autonomous dynamical systems", IEEE Transactions on Automatic Control, Vol. 33, No. 1, pp.16-27, Jan. 1988.

[6] Pavella M, Ernst D, and Ruiz-vega D. Transient stability of power systems: a unified Approach to assessment and control. Msssachusetts: Kluwer Academic Publishers, 2000.

[7] H. D. Chiang, F. F. Wu, P. P. Varaiya, "Foundation of direct methods for power systems transient stability analysis", IEEE Transactions on Circuits and systems, Vol. CAS-34, No.2, pp.160-173, Feb. 1987.

[8] R. J. Kaye, F. F. Wu, "Dynamic security regions of power systems," IEEE Transactions on Circuits \& Systems, Vol. CAS-29, No.9, pp.612$623,1982$.
[9] Y. X. Yu, "Security Region of bulk power system," Proceeding of the 2002 International Conference on Power System Technology, Kunming, China, Vol.1, pp.13-17, 2002.

[10] Y. X. Yu, W. P. Luan, "Practical Dynamic Security Regions of Power Systems," Proceedings of the Chinese Society for Electrical Engineering, Vol. 10, Supplement for Mathematicas of Elecrical Engineering, pp.22-28, Nov. 1990. (In Chinese)

[11] Y. Zeng, J. C. Fan, Y. X. Yu, et al., "Practical Dynamic Security regions of Bulk Power Systems," Automation of Electric Power Systems, Vol. 25, No.16, pp.6-10, 2001. (In Chinese)

[12] F. Feng, Y. X. Yu, "Dynamic security regions of power systems in injection spaces". Proceedings of the Chinese Society for Electrical Engineering, Vol. 13, No.3, pp.14-22, 1993. (In Chinese)

[13] Y. Zeng, Y. X. Yu, "A practical direct method for determining dynamic security regions of electrical power systems," Proceeding of the 2002 International Conference on Power System Technology, Kunming, China, Vol. 1, pp. 1270-1274, 2002.

[14] A. C. Xue, S. W. Mei, Q. Lu and Felix F. Wu. Approximation for the dynamic security region of network-reduction power systems. Automation of Electric Power systems, Vol.29, No.13, pp. 18-23, 2005.

[15] D. Z Cheng, J. Ma, "Calculation of stability region," in Proceedings. 2003 (42nd) IEEE Conference on Decision and Control, Vol. 6, pp. $5615-5620,2003$

[16] P. A. Cook and A. M. Eskicioglu, "Transient stability analysis of electric power systems by the method of tangent hypersurface", IEE Proceedings. Part C, Vol. 130, pp. 183-193, July 1983.

[17] P. M. Anderson and A. A. Fouad, Power System Control and Stability, The Iowa State University Press, Ames, Iowa, U.S.A. 1977.

\section{BIOGRAPHIES}

Ancheng Xue was born in Jiangsu, China, in Apr. 1979. He received his B. Sc degree in applied mathematics from the Dept. of Mathematics, Tsinghua University.. He is currently a Ph. D candidate in the Dept. of Electrical Engineering, Tsinghua Univ. His research interests include the nonlinear systems, control theory and their applications to the power systems.

Wei Hu received his B. Eng., M. Eng., and Dr. Eng. All from the Department of Electrical Engineering, Tsinghua University, Beijing, China. His research interest is in hybrid control theory applications in power systems.

Shengwei Mei (M'99) was born in Xinjiang, China, on Sept. 20, 1964. He received his B.S. degree in mathematics from Xinjiang University, M.S. degree in operations research from Tsinghua University, and Ph.D. degree in automatic control from Chinese Academy of Sciences, Beijing, in 1984, 1989, and 1996 respectively. He is now a professor of Tsinghua University. His current interest is in control theory applications in power systems.

Yixin Ni (SM'94) received her B. Eng., M. Eng., and Dr. Eng. All from the Department of Electrical Engineering, Tsinghua University, China in 1968, 1981 and 1983 respectively. Her research interests are power system stability and control, HVDC transmission, FACTS, and electricity markets. She was a professor of Tsinghua University and is now with the University of Hong Kong. She is a recipient of several nation-wide awards.

Felix F. Wu (SM'86, F'89) received his $\mathrm{Ph}$. D from University of California at Berkeley (UCB). He is currently Philip Wong Wilson Wong Professor in Electrical Engineering, at the University of Hong Kong, and Professor Emeritus, Department of Electrical Engineering and Computer Sciences at University of California, Berkeley.

Qiang Lu (SM'85, F'02) graduated from the Graduate School of Tsinghua University, China, in 1963 and joined the faculty of the same University. $\mathrm{He}$ was a visiting scholar and a visiting professor in Washington University, St. Louis and Colorado State University, Ft. Collins, respectively in 1984-1986 and a1993-1995. He is now a professor in Tsinghua University, and an academician of Chinese Academy of Science (1991). His research interest is in nonlinear control theory applications in power systems. 